University of Wollongong

Research Online

Faculty of Engineering and Information

Faculty of Engineering and Information

Sciences - Papers: Part A

Sciences

$1-1-2013$

\title{
An effective methodology to solve inverse kinematics of electroactive polymer actuators modelled as active and soft robotic structures
}

\author{
Rahim Mutlu \\ University of Wollongong, rm991@uowmail.edu.au \\ Gursel Alici \\ University of Wollongong, gursel@uow.edu.au \\ Weihua Li \\ University of Wollongong, weihuali@uow.edu.au
}

Follow this and additional works at: https://ro.uow.edu.au/eispapers

Part of the Engineering Commons, and the Science and Technology Studies Commons

Research Online is the open access institutional repository for the University of Wollongong. For further information contact the UOW Library: research-pubs@uow.edu.au 


\title{
An effective methodology to solve inverse kinematics of electroactive polymer actuators modelled as active and soft robotic structures
}

\author{
Abstract \\ Electroactive polymers (EAPs) generate highly non-linear deflections when they are used as actuators, \\ which are known as artificial muscles. Though several modelling methods have been proposed before to \\ understand their mechanical, chemical, electrical behaviours or 'electro-chemo-mechanical' behaviour, \\ estimating the whole shape deflection of the EAP actuators has not been studied yet. Therefore, we \\ report on (i) an effective methodology to estimate these actuators' whole shape deflection by employing a \\ soft robotic actuator/manipulator approach and (ii) an angle optimization method, which we call \\ AngleOPT, to accurately solve the EAP actuators' inverse kinematic problem. Laminated polypyrrole (PPy) \\ EAP actuators are employed to validate the soft robotic kinematic model which has more degrees of \\ freedom than its input. This follows that we have reduced a difficult problem to an easy-to solve inverse \\ kinematic problem (easier to solve) of a hyper-redundant soft robotic system. A parametric estimation \\ model is also proposed to predict the tip coordinates of the actuators for a given voltage. The \\ experimental and numerical results are presented to demonstrate the efficacy of the methodology for \\ estimating the EAP actuators' highly non-linear bending behaviour from the inverse kinematic model. The \\ proposed methodology can be extended to other type of smart structures with a similar topology. (C) 2013 \\ Elsevier Ltd.

\section{Keywords} \\ polymer, actuators, modelled, active, electroactive, soft, effective, robotic, structures, kinematics, inverse, \\ solve, methodology

\section{Disciplines} \\ Engineering | Science and Technology Studies

\section{Publication Details} \\ Mutlu, R., Alici, G. \& Li, W. (2013). An effective methodology to solve inverse kinematics of electroactive \\ polymer actuators modelled as active and soft robotic structures. Mechanism and Machine Theory, 67 \\ 94-110.
}




\title{
AN EFFECTIVE METHODOLOGY TO SOLVE INVERSE KINEMATICS OF ELECTROACTIVE POLYMER ACTUATORS MODELLED AS ACTIVE AND SOFT ROBOTIC STRUCTURES
}

\author{
Rahim Mutlua, Gursel Alici, ${ }^{\mathrm{a}, \mathrm{b}}$ and Weihua $\mathrm{Li}^{\mathrm{a}}$ \\ ${ }^{a}$ School of Mechanical, Materials and Mechatronic Engineering \\ ${ }^{\mathrm{b}} \mathrm{ARC}$ Centre of Excellence for Electromaterials Science \\ University of Wollongong, NSW, 2522, Australia
}

Corresponding Author: Gursel Alici (ph: +6142214115, fax: +61242215474, gursel@uow.edu.au)

\begin{abstract}
Electroactive polymers (EAPS) generate highly non-linear deflections when they are used as actuators, which are known as artificial muscles. Though several modelling methods have been proposed before to understand their mechanical, chemical, electrical behaviours or 'electro-chemo-mechanical' behaviour, estimating the whole shape deflection of the EAP actuators has not been studied yet. Therefore, we report on (i) an effective methodology to estimate these actuators' whole shape deflection by employing a soft robotic actuator/manipulator approach and (ii) an angle optimization method, which we call AngleOPT, to accurately solve the EAP actuators' inverse kinematic problem. Laminated polypyrrole (PPy) EAP actuators are employed to validate the soft robotic kinematic model which has more degrees of freedom than its input. This follows that we have reduced a difficult problem to an easy-to solve inverse kinematic problem (easier to solve) of a hyper-redundant soft robotic system. A parametric estimation model is also proposed to predict the tip coordinates of the actuators for a given voltage. The experimental and numerical results are presented to demonstrate the efficacy of the methodology for estimating the EAP actuators' highly non-linear bending behaviour from the inverse kinematic model. The proposed methodology can be extended to other type of smart structures with a similar topology.
\end{abstract}

Keywords: Electroactive polymer actuators, soft robotics, inverse kinematics, nonlinear constrained optimization, smart structures 


\section{INTRODUCTION}

Electroactive polymers (EAPs) have been receiving the attention of researchers since the mid-1970s. They are usually tailored as actuators (i.e. also known as artificial muscles) and sensors [1]. The EAP actuators are considered to be alternatives to conventional actuation means due to their specific characteristics including compliance, low electrical energy consumption, suitability to miniaturization, biocompatibility, operation ability in air and aqueous environments, and a high force output to weight ratio. Although the EAP actuators have a few configurations, one configuration receiving significant attention recently is a multilayer laminated-configuration. A multi-layer laminated EAP actuator consists of a passive layer as a cell separator and active polymer layers grown on both sides of the passive layer as electrodes. Such actuators can operate in dry environments as opposed to bi-layer ionic EAP actuators, which have to operate in a certain aqueous electrolyte. In this study, we use the multi-layer laminated ionic EAP actuators. When an electrical input is applied across the two polymer layers, they change their volume due to the movement of electrolytic ions (anions and cations) in and out of the electroactive polymer layers. This volume change generates a mechanical output, in the form of a bending displacement and/or a blocking force when these actuators are configured like a cantilevered beam. Besides tailoring the EAPs as actuators, they can be used as sensors; when a mechanical displacement or force is applied, they generate an electrical signal indicating the output [2].

Many applications based on the EAP actuators have been proposed before. These potential applications include swimming devices, crawling robots, micro manipulators, robotic grippers, motion converter mechanisms and many more [3-8]. The EAP actuators are especially suitable for biologically-inspired robotics for which actuators mimicking the characteristics of natural muscles are needed. For instance, these actuators were successfully demonstrated in fish swimming as artificial muscles articulating a caudal fin for propulsion [4].

In addition to utilizing the EAP actuators as artificial muscles in such bio-inspired robotic systems, for more advanced applications, a complete model is required to predict their whole shape behaviour in real time under an electrical input or under a displacement or a force input if the electroactive polymer is used as a sensor. 
Though there are some significant studies on modelling EAP actuators [8-13, 27], majority of them focus on modelling the actuator as a cantilever beam and predicts its tip displacement rather than its whole shape or overall configuration. These studies generally follow a linear deflection principle based on small deflections not higher than $20 \%$ of the actuator length. However, these actuators can deflect as high as $50 \%$ of the actuator length. With this in mind, there is a need for a more accurate approach to estimate the tip deflection and the overall shape or configuration of these active and soft robotic structures.

Soft robotics, which require soft and hyper flexible components to build a soft robotic device, is an emerging robotics field. Several studies have been conducted on soft and hyper-redundant actuators and manipulators built with conventional materials and components. Whether the soft robotic structure is an actuator or a manipulator with many DoFs, their kinematic model is developed following the same methodology. While a soft robotic manipulator is a continuum system, a hyperredundant manipulator can be modelled as a discrete system consisting of serially connected rigid links. When the number of links in the manipulator is high enough and link lengths are small enough, the manipulator can be treated as a soft robotic manipulator. There are some studies on such robotic systems inspired from nature in which the working mechanisms of an octopus arm, an eel [14, 15], an elephant trunk [16-17] and a snake [20] are mimicked. Following similar modelling principles, Suzumori et al. developed a passive flexible micro-actuator fabricated from a fibre reinforced rubber which is powered by air pressure [19]. These devices are built by conventional actuation means such as tendons, pneumatic actuators, servo motors and joints [20] or a passive material such as a reinforced rubber [16, 17, 19]. In the literature, kinematic models are established for such soft and hyper-redundant robotic systems using a so-called backbone curve approach [20-24]. The backbone curve approach assumes that the posture of the manipulator is represented by a spatial curve (backbone) and the links of the whole manipulator are fully oriented along (or tangent to) this curve. Formulating the backbone curve of the soft robotic structure is straight forward for a continuum hyper-redundant system. However, obtaining inverse kinematic and/or dynamic solutions of a soft robotic structure can be difficult. In the literature, there are several methodologies such as modal based approach [20, 21, 24], optimization [22, 23] and tractrix based approaches [25, 26] to 
solve the inverse kinematic problem of soft hyper-redundant robot manipulators or actuators. These methodologies can be extended to soft manipulation systems based on EAP actuators.

This paper presents an effective methodology to model and solve the inverse kinematics of a bending type EAP actuator which is treated as an active and soft robotic manipulator with a very large kinematic redundancy. We employ an optimization method (AngleOPT) in order to solve the hyper-redundant inverse kinematic model. We have also employed an image processing technique to measure the tip position of the PPy-EAP actuator as a function of time and used these data as the input to the hyper-redundant inverse kinematic model in order to estimate the angular position, velocity and acceleration (i.e., joint variables) of each segment or each link forming the soft robotic structure (the PPy-EAP actuator). After demonstrating the capability of the AngleOPT for estimating the joint variables, a parametric model is also established in order to obtain a relation between the electrical input (i.e. voltage) and the EAP actuators' tip coordinates as a function of time. This parametric model is then employed to calculate the tip coordinates of the actuators for the whole shape estimation algorithm (the AngleOPT). The parametric model can be used to estimate the tip coordinates when it is not possible to measure or retrieve the tip coordinates of a given actuator for the shape estimation algorithm. The parametric model complements the inverse kinematic algorithm in estimating the whole configurations of a given actuator as a function time.

The primary contribution of this study is to propose an effective methodology for modelling the kinematic behaviour the EAP actuators and employing a constrained optimization method to solve the hyper-redundant inverse kinematic model such that the position of each link in the hyper-redundant structure is physically compatible with the overall shape of the EAP actuator. It must be noted that we have modelled the EAP actuator as an active and soft robotic structure with hyper-redundant degrees of freedom. When solving its hyper-redundant inverse kinematic model, the multiple solutions for the inner links are eliminated by specifying proper upper and lower boundaries and constraints for the joint angular positions reflecting the physical configuration of the actuator during operation. The same controllability over the joint positions, and therefore over the whole shape of the soft robotic structure cannot be achieved with an analytical method or numerical techniques based on 
inverting the manipulator's Jacobian matrix (pseudo-inverse as the EAP actuator is modelled as a hyper-redundant system).

\section{ELECTROACTIVE POLYMERS: Fabrication \& Operation Principles}

The electroactive polymers are smart materials derived from monomers; commonly from pyrrole, thiophene and aniline. They have been used to establish various devices including sensors, membranes and materials for energy storage and actuators [2-7].

In this paper, the multi-layer laminated electroactive polymer actuator type is used. Pyrrole monomer is used to fabricate the EAP actuator's active polymer layers by following a number of steps. Firstly, both sides of a non-conductive porous layer (i.e. polyvinylidene fluoride, PVDF) were sputter coated with a thin gold layer to obtain a conductive substrate with a resistance as low as $20 \Omega$. The PVDF layer acts as an electrochemical cell separator and also stores electrolytic ions (e.g., Li.TFSI). The PVDF layer used in this study is $110 \mu \mathrm{m}$ in thickness as received. Meanwhile, a polymer growth solution containing pyrrole monomer $(0.1 \mathrm{M})$, lithium triflouromethanesulfonimide $\left(\mathrm{Li}^{+} \mathrm{TFSI}^{-}, 0.1 \mathrm{M}\right)$ and $1 \%$ water in propylene carbonate (PC) was prepared for the polymerisation process. Then the gold coated PVDF was submerged in this solution using supports to prevent any short circuit between a working electrode (the gold coated PVDF) and a counter electrode. The polypyrrole (PPy) layers were galvanostatically grown from the growth solution at a current density of $0.1 \mathrm{mAcm}^{-2}$ for 12 hours on the gold coated PVDF. This polymerisation process provides $\sim 30 \mu \mathrm{m}$ thickness of PPy layer on both sides of the gold coated PVDF. The total thickness of the laminated PPy based EAP actuator was approximately $170 \mu \mathrm{m}$. The laminated polypyrrole based electroactive polymer actuator is called PPy-EAP actuator throughout the paper unless otherwise stated. The PPy-EAP actuator's laminated (tri-layer) configuration and operation principle are depicted in Fig.1.

The PPy-EAP actuator's operation principle is based on the energy conversion from an electrochemical process to a mechanical output. An electrical input applied to the polymer layers stimulates counter-ions to move in and out of the PPy layers. The $\mathrm{TFSI}^{-}$anions move from the electrolyte stored in the PVDF layer into the positively charged PPy layer and an opposite reaction happens in the other PPy layer in order 
to neutralize the charge in the PPy layers. This ion movement causes a volume expansion in the positively charged PPy layer and a volume contraction in the other PPy layer. This electro-chemo-mechanical process thus generates a bending behaviour in the PPy-EAP actuator, as illustrated in Fig.1.

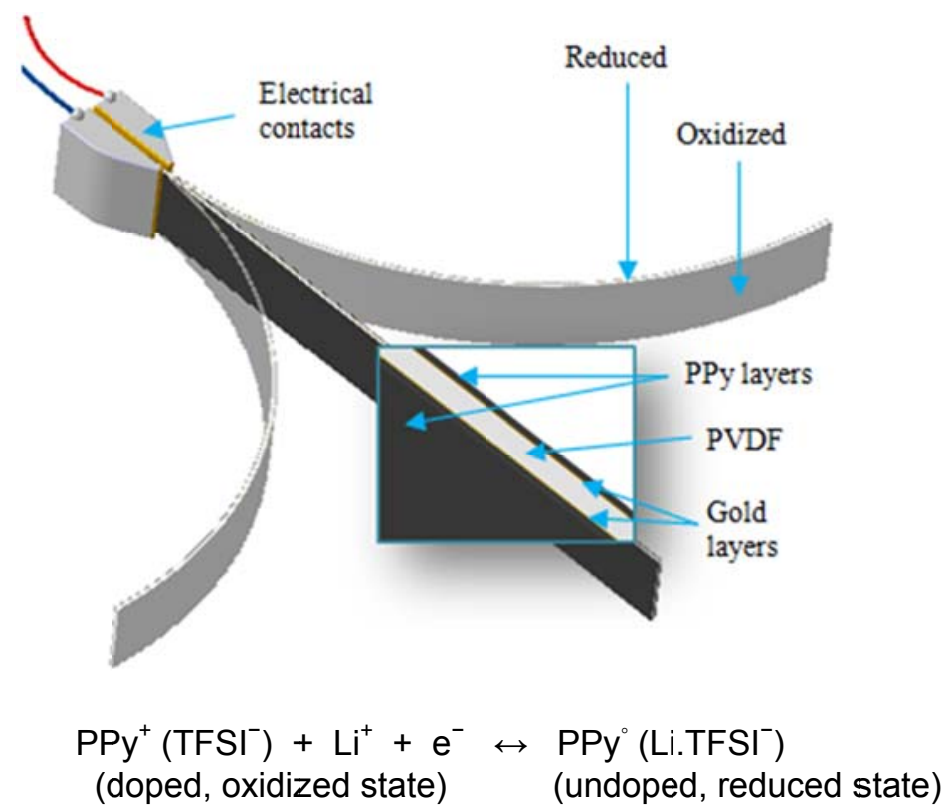

Fig.1. Structure and the operation principle of the laminated PPy-EAP actuator

\section{KINEMATIC ANALYSIS OF THE EAPS: A Soft Robotic Modelling Approach}

The bending behaviour of the EAP actuator shown in Fig. 1 can be estimated by employing the classical beam theories such as Euler-Bernoulli and Timoshenko beam theories [12]. However, these theories assume that the beam material has a constant modulus of elasticity and the beam shows a linear deflection behaviour such that the longitudinal deflection of the beam is negligibly small and the traverse deflection is less than $20 \%$ of the beam length [12]. The EAP actuators not only generate non-linear and very large deflections, but also have condition-dependent properties (e.g. Modulus of elasticity of the EAP actuator changes as a function of the electrical stimulus applied to the EAP actuator). Therefore, the classical beam theories fail to estimate the highly non-linear deflection of the EAP actuators. For a given actuator tip deflections $(X, Y)$, it is challenging to estimate the whole shape of the EAP actuator as a continuum structure especially for medical applications such as using it as a catheter. We employ the backbone curve approach to obtain the hyper-redundant kinematic model of the PPy-EAP actuator. 
The backbone curve approach assumes that a special curve called the backbone curve passes through the geometric centre of each link of the manipulator. Previously, we employed this approach to model the kinematics of a PPy-EAP actuator generating a lamina emergent 3D motion from its initial planar configuration [28]. The kinematic model of the PPy-EAP actuator is comprised of two steps;

1. Obtain a parameterized function to describe the PPy-EAP actuator's backbone curve,

2. Do the link alignments by generating and orientating the cross sections of the PPy-EAP actuator utilizing Frenet formulations and Denavit-Hartenberg transformations assuming that the cross sections are always perpendicular to the backbone curve.

Fig.2 illustrates the PPy-EAP actuator's soft robotic manipulator representation and the parameters describing the backbone curve.

The backbone curve of the PPy-EAP actuator can be defined, in Euclidean space with respect to its origin at the fixed end of the actuator, as

$$
\boldsymbol{r}(\sigma, t)=\left[r_{x}(\sigma, t), r_{y}(\sigma, t), r_{z}(\sigma, t)\right]^{T}
$$

where $\boldsymbol{r}(\sigma, t):[0, L] \rightarrow \mathbb{R}^{3}$ assigns a position in the Euclidean space to each link parameter $\sigma \in[0, L]$ in time $t[30] . L$ is the overall length of the backbone curve.

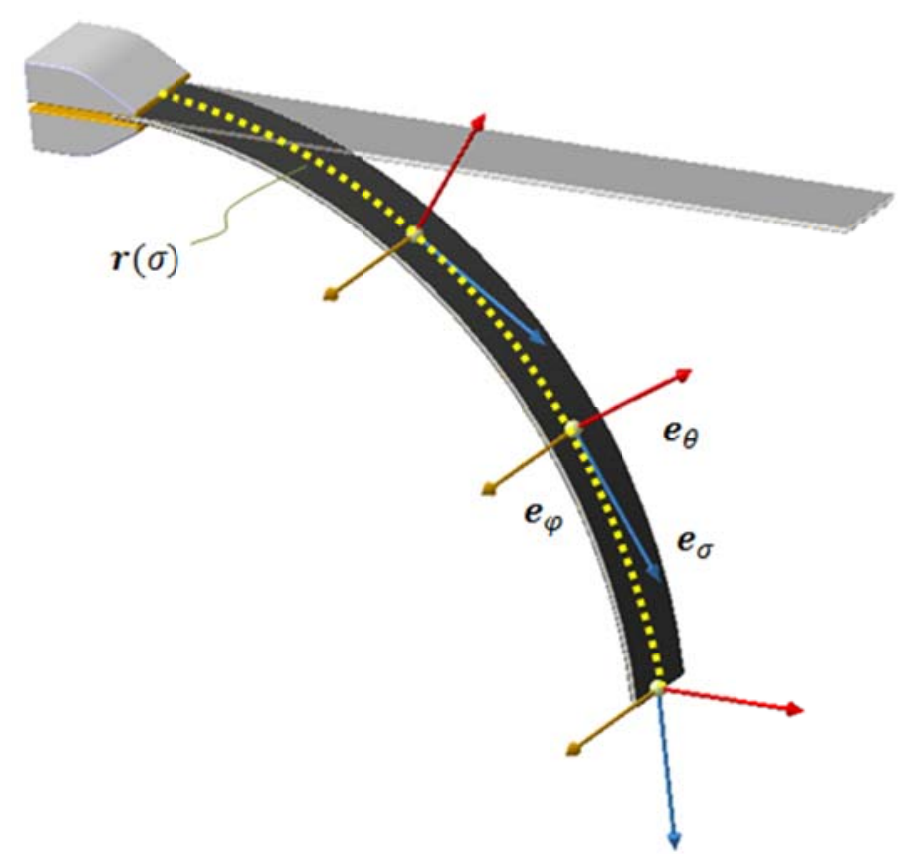

Fig.2. The configuration of the PPy-EAP actuator is defined by its backbone curve, $\boldsymbol{r}(\sigma)$ 
The position vectors along each axis of the link parameter $\sigma$ are described as

$$
\begin{aligned}
& r_{x}(\sigma, t)=\{l \cos \theta \sin \varphi\}_{\sigma}+r_{x}(\sigma-1, t) \\
& r_{y}(\sigma, t)=\{l \sin \theta \sin \varphi\}_{\sigma}+r_{y}(\sigma-1, t) \\
& r_{z}(\sigma, t)=\{l \sin \varphi\}_{\sigma}+r_{z}(\sigma-1, t)
\end{aligned}
$$

where $l$ is the length of each link. Each position vector is described relative to the previous Frenet frame. Frenet frames also play a key role in calculating the orientations of the discretised sections of the PPy-EAP actuator's continuum structure. The whole shape of the PPy-EAP actuator can be obtained by integrating its backbone curve over the link parameter (differential increments, $d \sigma$ if the PPyEAP actuator is modelled as a continuum system) from the fixed end to the free end of the actuator or summing up the position vectors if the PPy-EAP actuator is modelled as a hyper-redundant manipulator. The local coordinate frame (Frenet frame) of each link $\boldsymbol{E}(t):\left[\begin{array}{lll}\boldsymbol{e}_{\sigma} & \boldsymbol{e}_{\theta} & \boldsymbol{e}_{\varphi}\end{array}\right]$ is described by the three orthonormal vectors;

$$
\begin{aligned}
& \mathrm{e}_{\sigma}=\mathrm{dr} / \mathrm{d} \sigma \\
& \mathrm{e}_{\theta}=\left(\mathrm{de}_{\sigma} / \mathrm{d} \sigma\right) /\left\|\left(\mathrm{de}_{\sigma} / \mathrm{d} \sigma\right)\right\| \\
& \mathrm{e}_{\varphi}=\mathrm{e}_{\sigma} \times \mathrm{e}_{\theta}
\end{aligned}
$$

This Frenet formulation describes the motion of this orthogonal local frame along the backbone curve. The formulas for this motion are given as

$$
\frac{d t}{d s}=\kappa n, \quad \frac{d n}{d s}=-\kappa n+\tau b, \quad \frac{d b}{d s}=-\tau b
$$

where $\kappa$ and $\tau$ are the curvature and torsion of the backbone curve, respectively.

Since the PPy-EAP actuator is cantilevered, the bending motion of the EAP actuator happens in $2 \mathrm{D}$ plane. This reduces the backbone curve of the actuator to

$$
\boldsymbol{r}(\sigma, t)=\left[r_{x}(\sigma, t), r_{y}(\sigma, t)\right]^{T}
$$

The backbone of the PPy-EAP actuator in 2-dimensional Euclidean space and its position vectors are illustrated in Fig.3. It must be noted that the soft robotic 
kinematic model using backbone curve approach has been set up for a general case (i.e. in 3D). This general kinematic model has then been reduced to a $2 \mathrm{D}$ model that describes the PPy-EAP actuator's bending behaviour.

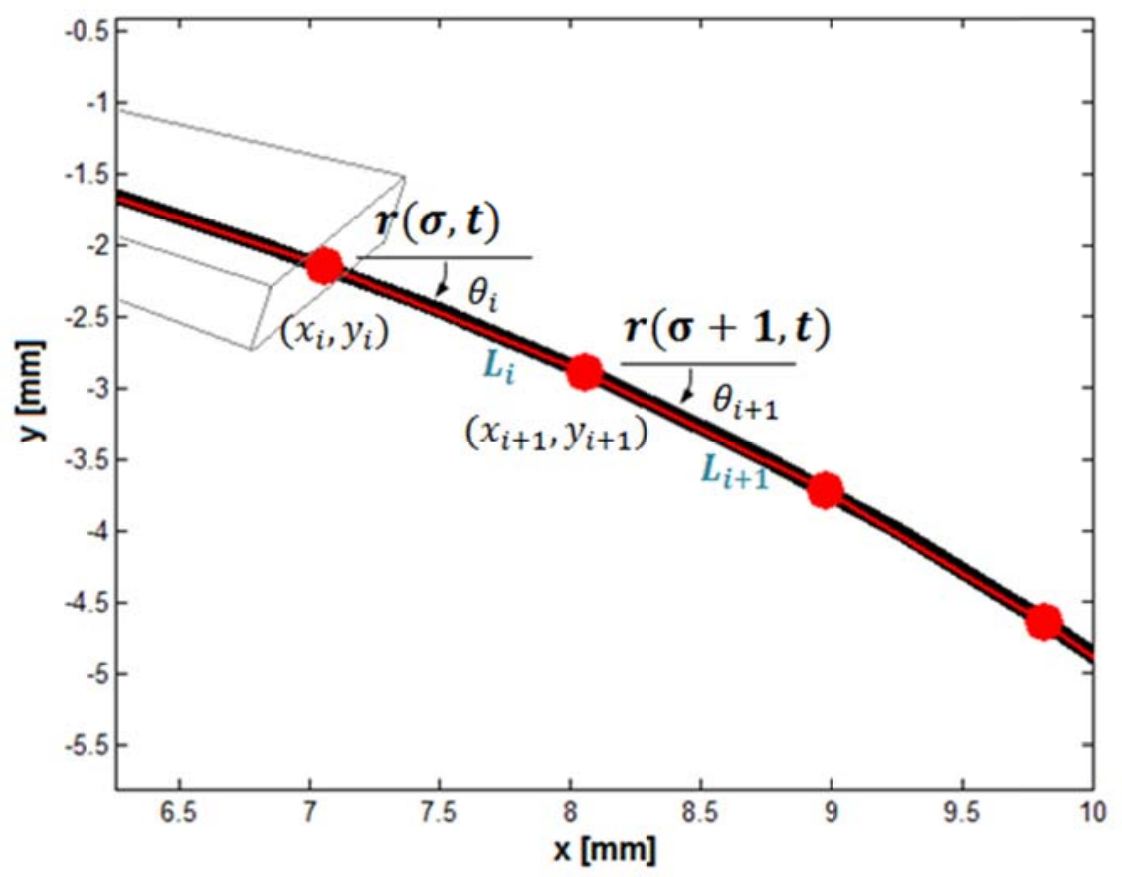

Fig.3. Position vectors of the PPy-EAP actuator model.

After obtaining the analytical expressions for the backbone curve (Eq.11) which represents the forward kinematic model of the PPy-EAP actuator, we construct a constrained-optimization function, as presented in the next subsection. The solutions (joint angles) of this function are the inverse kinematic solution of the PPy-EAP actuator, from which we can estimate its whole continuum structure for a given tip coordinate $(X, Y)$.

\subsection{Optimization Method, AngleOPT, to Solve Inverse Kinematics}

Inverse kinematics is one of the major problems in robotics due to the existence of multiple solutions or configurations for a given manipulator position in the Cartesian space. Though an analytical solution can be obtained for less than 3-DoF manipulators, it is virtually impossible to obtain an analytical inverse kinematic solution for high DoF manipulators (i.e. hyper-redundant robotic manipulators). With this in mind, numerical methods can be employed to obtain the inverse kinematic variables; joint angles, velocities and accelerations. Methods such as Jacobian matrix and least square estimations are the possible methods to calculate the inverse kinematic variables. However, an optimization approach, which we call the 
AngleOPT, simplifies the complexity of the inverse kinematic problem. It does not require mathematical manipulations prior to computing the inverse kinematic variables, provided that appropriate joint constraints are imposed on the inverse kinematic solutions. In other words, we formulate the hyper-redundant inverse kinematic problem of the PPy-EAP actuators as a constrained optimization problem. The AngleOPT approach reported in this paper is solved by employing fmincon function of MATLAB which uses the Sequential Quadratic Programming (SQP) algorithm. Although the SQP is relatively easy to implement, this algorithm does not necessarily find global minimum(s) unless initial values of the variables and constraints for these variables are selected based on the physical configuration of the manipulator. If the function ' $f$ ' represents the hyper-redundant kinematic model of the soft robotic structure, the joint variables can be found by minimizing the function ' $f$ ' with a set of local constraints, as presented below;

$$
\min _{\theta} f(l, \theta, X, Y)
$$

i. $\quad$ lower and upper boundaries

ii. constraints between and for joint angles

$$
\begin{aligned}
& l b \leq \theta_{i} \leq u b \\
& A \cdot \theta \leq b
\end{aligned}
$$

where $l$ is the length of the individual links, $\theta$ is the joint angle in xy-plane, $X$ and $Y$ are the tip coordinates of the PPy-EAP actuator relative to the fixed (base) point of the PPy-EAP actuator, $l b$ and $u b$ are the lower and upper boundaries for the joint angles, respectively. $A \cdot \theta \leq b$ are the constrained relations for the joint angles. It must be noted that a joint angle closer to the free end of the PPy-EAP actuator should be larger than the joint angles closer to the fixed end. This follows that the constraints should reflect the physical configuration of the actuator. To do that, we propose an adaptive method for specifying the constraint relations of the PPy-EAP actuator. The constraints are updated for each configuration of the actuator as a function of time. Further details on the implementation of the AngleOPT are provided in Section 4.2.

\subsection{Comparison of AngleOPT Approach with Jacobian and Analytical Methods}


An analytical solution of the inverse kinematic problem is the ideal method to determine the kinematic configurations of a robot manipulator for a given tip position. However, analytical methods are limited to low degree-of-freedom manipulators. In other words, it is virtually impossible to establish an analytical inverse kinematic model for a hyper-redundant manipulator. Further, it is not possible to determine the manipulator configurations with a smooth transition from one link to another such that all links of the manipulator form a smooth continuum manipulator. The higher is the number of links of the manipulator, the higher is the number of kinematic configurations. Even though the coordinates of both ends of the EAP actuator are known, many different kinematic configurations satisfying these boundary conditions can be obtained. In order to estimate the exact kinematic configurations of an active soft structure such as EAP actuators, analytical joint constraints are required if the active soft structure is modelled as a hyper-redundant system. This is a time consuming and tedious task requiring a higher computation power.

The Jacobian method generates solutions for a given configuration of a manipulator with an error accumulation from the first configuration to the next configuration. This follows that the higher is the number of configurations, the higher is the error associated with the final configuration. The Jacobian method also requires additional mathematical manipulations and constraints in order to have a control on the estimated the backbone curve/shape of the PPy-EAP actuator. More importantly, the Jacobian-matrix based methods can be numerically unstable when approaching a kinematically singular configuration [29]. Further, for a hyper-redundant system, the Jacobian matrix is not square and therefore, obtaining its inverse is problematic and introduces further numerical inaccuracies due to the inversion of a non-square matrix. The Jacobian method also requires finer increments from one configuration to another, requiring a relatively higher computation power or time. On the other hand, the AngleOPT, as an optimization based method, calculates joint angles for every configuration with no error accumulation. Also, the AngleOPT method does not require small incremental changes of the configuration as it searches for the joint angles for a given configuration. More importantly, the AngleOPT method allows the accurate shape estimation of the manipulator such that it finds the joint angles reflecting the continuum structure of the PPy-EAP actuator.

\subsubsection{A 2-DoF model representation}


A two link manipulator is used to compare the AngleOPT method with the analytical method and the Jacobian method. Fig.4 shows the 2-link representation of the PPyEAP actuator, as a rough approximation.

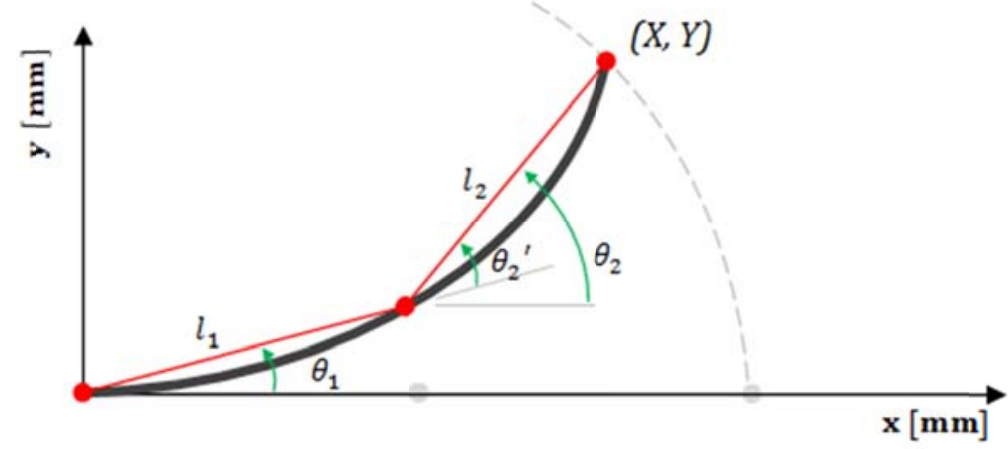

Fig.4. 2-rigid-link (red lines) representation of the PPy-EAP actuator

The analytical inverse kinematic equations (Eqs.13-15) for a 2-link model with revolute joints are available in [31]. The length of the PPy-EAP actuator used for this case is $20 \mathrm{~mm}, 10 \mathrm{~mm}$ for each link in the 2-link model. The accuracy of the 2-link inverse kinematic model is evaluated by calculating the error between the given tip coordinates and the tip coordinates obtained from the inverse kinematic solutions. Fig. 5 shows the errors from the three methods. The analytical method error is primarily due to truncations and round-offs in the inverse kinematic calculations. Taking the analytical method as a reference, the AngleOPT method shows quite satisfactory results with no-error accumulation. On the other hand, the Jacobian method gives much higher errors even for a simple 2-link model. Also, it must be noted that, for this 2-link model example, small incremental changes of the tip coordinates are ensured not to cause any numerical instability issues for the Jacobian method.

$$
\begin{gathered}
\theta_{2}^{\prime}=\cos ^{-1}\left(\frac{X^{2}+Y^{2}-\left(l_{1}^{2}+l_{2}^{2}\right)}{2 l_{1} l_{2}}\right) \\
\theta_{1}=-\sin ^{-1}\left(\frac{l_{2} \sin \theta_{2}{ }^{\prime}}{\sqrt{X^{2}+Y^{2}}}\right)+\operatorname{Atan} 2(Y, X) \\
\theta_{2}=\theta_{1}+\theta_{2}{ }^{\prime}
\end{gathered}
$$



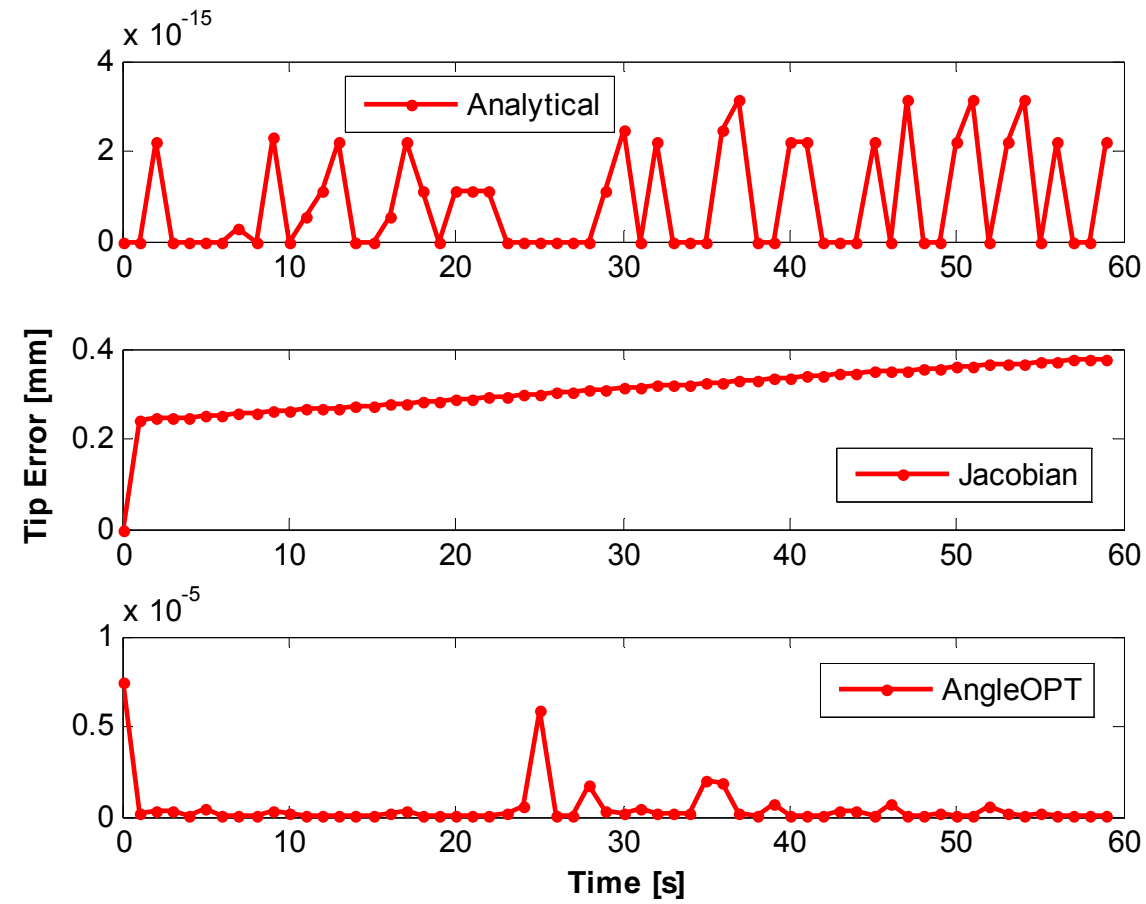

Fig.5. Tip error comparison between the analytical, the Jacobian and the AngleOPT methods. There is an error accumulation in the Jacobian solution as it is based on previous kinematic calculations

\section{EXPERIMENTAL RESULTS TO VERIFY INVERSE KINEMATIC MODEL}

Experiments were conducted to obtain the PPy-EAP actuator's tip displacement for the electrical input ranging from 0.25 to $1.0 \mathrm{~V}$. We employed the actuation and image processing systems depicted in Fig.6. The electrical input signals were generated using a SIMULINK program, and passed through an USB-type NI-DAQ card (NI USB-6251) to a potentiostat. The electrical input supplied from the potentiostat was applied to the PPy-EAP actuator using a gold coated clamp. The motion of the PPyEAP actuators was recorded using a digital camera (Nikon D5100). The videos were recorded at 30fps which was sufficient to record the bending motion of the PPy-EAP actuators. The PPy-EAP actuators were stimulated for $\sim 40$ seconds depending on the electrical input (step input) -to cover the total bending range of the PPy-EAP actuator; from its neutral (the actuator is straight and not activated) to its fully charged (maximum bent) state. After recording the PPy-EAP actuators' bending motion under different electrical inputs, the recorded videos were analysed to determine the tip displacement data by an image processing algorithm. The whole experimental setup including the actuation of the PPy-EAP actuators, the image processing and the hyper-redundant inverse kinematic solution algorithms is 
illustrated in Fig.6. The PPy-EAP actuators used in the experiments had the same dimensions, $20 \times 3 \times 0.17 \mathrm{~mm}$, length, width and thickness, respectively.

The accuracy of the hyper-redundant inverse model and its solution is evaluated by calculating the root means square (RMS) of the error. In this case, the RMS is applied to the error between the experimental tip displacement data extracted from the images and the tip data re-generated from the joint angles estimated by the AngleOPT and the Jacobian methods. The RMS error for the tip data is calculated from

$$
\begin{aligned}
& R M S_{x}=\sqrt{\frac{1}{n} \sum_{j=1}^{n}\left(x_{r}-x_{e}\right)_{i}^{2}} \\
& R M S_{y}=\sqrt{\frac{1}{n} \sum_{j=1}^{n}\left(y_{r}-y_{e}\right)_{i}^{2}}
\end{aligned}
$$

\subsection{Image Processing}

The image processing algorithm used in this study consists of two steps; i) generating images from the video recorded, ii) extracting tip coordinates $(X, Y)$ from the images. The images were extracted in every second or half a second depending on the duration of the bending motion of the PPy-EAP actuator and frequency of the electrical input. After the image extraction from the videos recorded, another program was employed to obtain the tip displacement data $(X, Y)$ of the actuator. These tip coordinates are the inputs for the inverse kinematic model to predict the whole configuration of the PPy-EAP actuator from its no-input state to the fully charged state. 


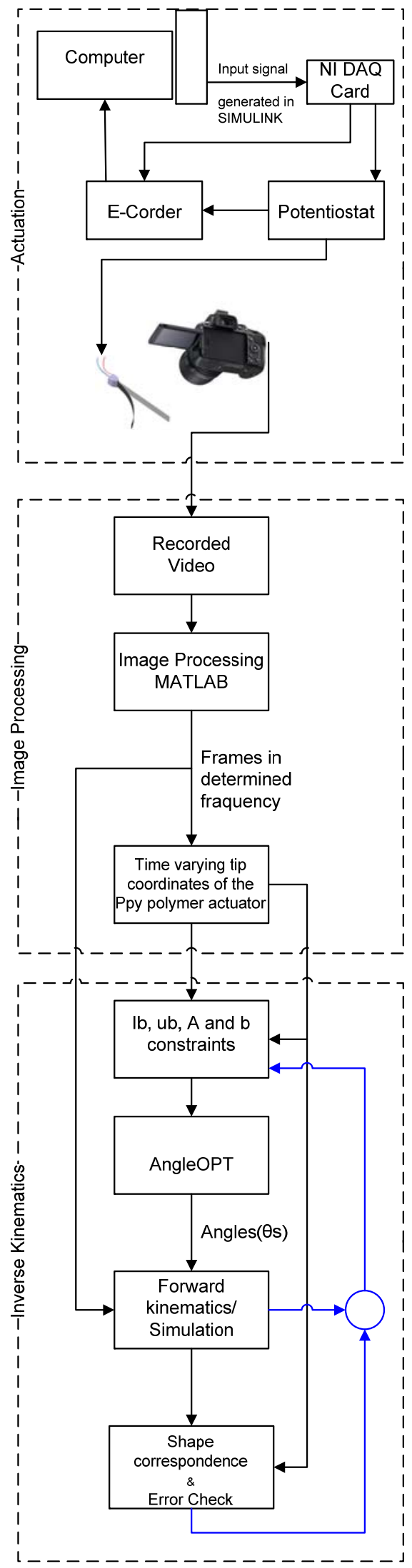

Fig.6. The schematics of the experimental system and the inverse kinematic solution method for the PPy-EAP actuator 


\subsection{Solution of a 16-DoF Model and Its Boundary/Constraint Specifications}

As stated before, the PPy-EAP actuator can be modelled as a soft robotic structure with a high degree of redundancy. Here we model it as a 16-DoF hyper-redundant system with the optimization function in Eq.18 in order to calculate the angular positions of its 16 joints. For the tip coordinates $(X, Y)$ of the PPy-EAP actuator, the optimization function is constructed as

$$
\min _{\theta}\left(f=\left[\begin{array}{c}
L_{1} \cos \theta_{1}+L_{2} \cos \theta_{2}+\ldots+L_{n} \cos \theta_{n} \\
L_{1} \sin \theta_{1}+L_{2} \sin \theta_{2}+\ldots+L_{n} \sin \theta_{n}
\end{array}\right]-\left[\begin{array}{c}
X \\
Y
\end{array}\right]\right) \quad n=16
$$

It must be noted that the number of degrees of freedom is chosen 16 here for the sake of convenience. Any appropriate high DoF can be considered similarly -- the higher is the DoF, the more accurate is the configuration estimation. The joint angles describing the link positions should be such that there is a smooth transition from one link to the next to accurately match the physical configuration of the actuator. This cannot be demonstrated with a 2-DoF model presented in Section 3.3.1. We have employed the AngleOPT method to solve the inverse kinematic problem described here in order to find the joint positions of the 16-DoF PPy-EAP actuator. The joint positions calculated will generate a shape correspondence between the real PPy-EAP actuator and the 16-DoF hyper-redundant model in real time.

To obtain the joint positions compatible with the physical configuration of the polymer actuator, we set joint boundaries and constraints. To this aim, we introduce an adaptive constraint speciation approach to have an accurate shape correspondence between the real PPy-EAP actuator and the instantaneous solutions obtained from the 16-DoF hyper-redundant model. The adaptive constraint speciation approach updates the constraints and boundaries instantaneously for every configuration of the actuator estimated from the hyper-redundant model. For instance, the joint angles (bending angles) of the links of the hyper-redundant model should have an increasing trend from the first link to the last link which is the free end of the PPyEAP actuator; the joints closer to the free end of the PPy-EAP actuator should have a relatively higher deflection angle. Further, the joint angles closer to the free end of the actuator have a larger change (a higher angular velocity) which should be 
reflected on the constraints and boundaries. Once the proper physical parameters (i.e. link lengths) and the constraints and boundaries are assigned to the 16-DoF inverse kinematic model, the AngleOPT determines the joint angles describing the overall configuration of the actuator or the manipulator as a continuum system. The assigned constraints/relations used for solving the inverse kinematics of the 16-DoF hyper-redundant actuator are given in Appendix A.

The transient configurations of the 16-DoF hyper-redundant model and the real PPyEAP actuator are shown in Figures 7-10 which show the motion profiles of the real actuator and the model actuator from the neutral state (aligned with the y-axis) to the final state, and the local coordinate frames (i.e. Frenet frames) orientated along the PPy-EAP actuator's estimated shape. As mentioned above, the local coordinates do not play a significant role in $2 \mathrm{D}$ inverse kinematic shape estimation of the PPy-EAP actuator, but they are used to calculate curvature of the PPy-EAP actuator and orientate discretised sections along the estimated shape. Figures 7-10 show the PPy-EAP actuator's actuation cycle under $0.25,0.50,0.75$ and $1.0 \mathrm{~V}$ electrical inputs, respectively.

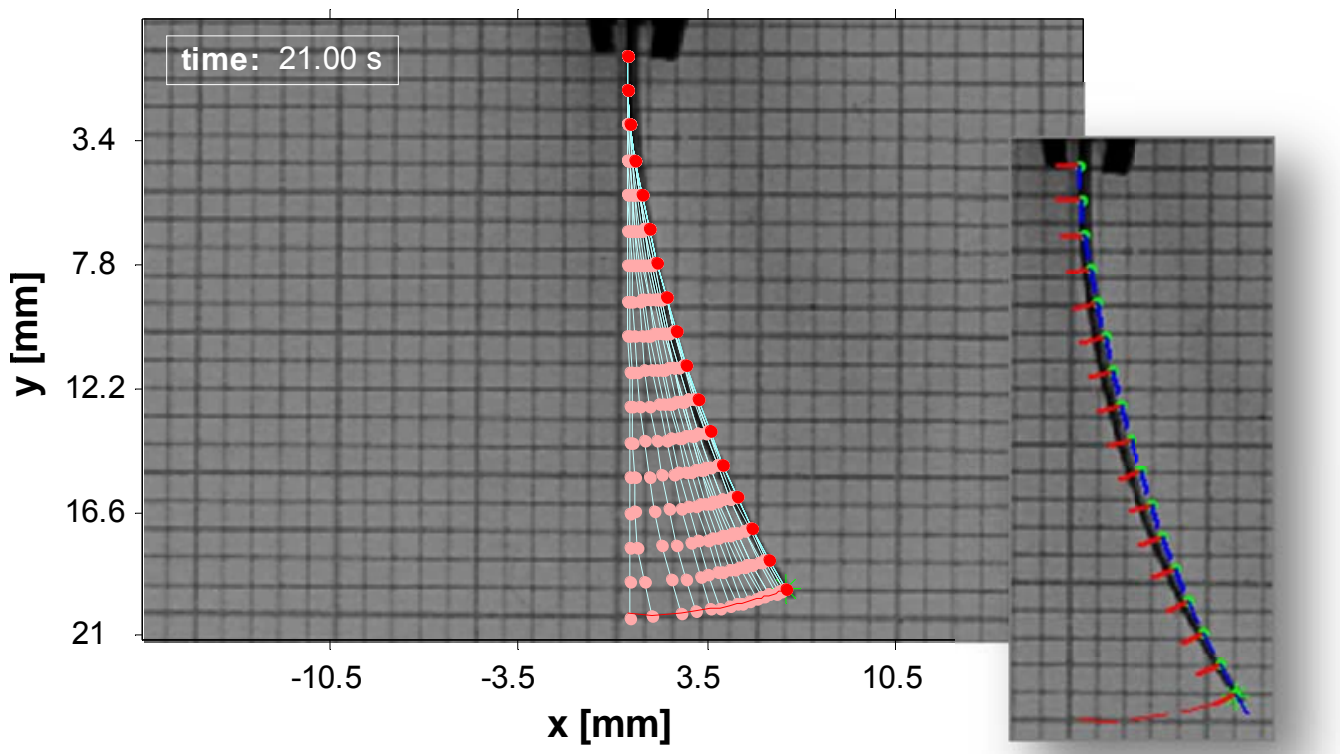

Fig.7. Shape correspondence between the 16-DoF hyper redundant model (light green - pink dotted line shows the sequences of the model's configuration, and light green - red dotted line shows the final configuration) and the PPy-EAP actuator (thick black curve as it is on the background of the figure / in its final configuration) under $0.25 \mathrm{~V}$. Red line is the experimentally obtained tip path. (The final configuration of both the PPy-EAP actuator and the 16-DoF hyper-redundant model are shown in the inner image on the right with reference frames) (Color print). 


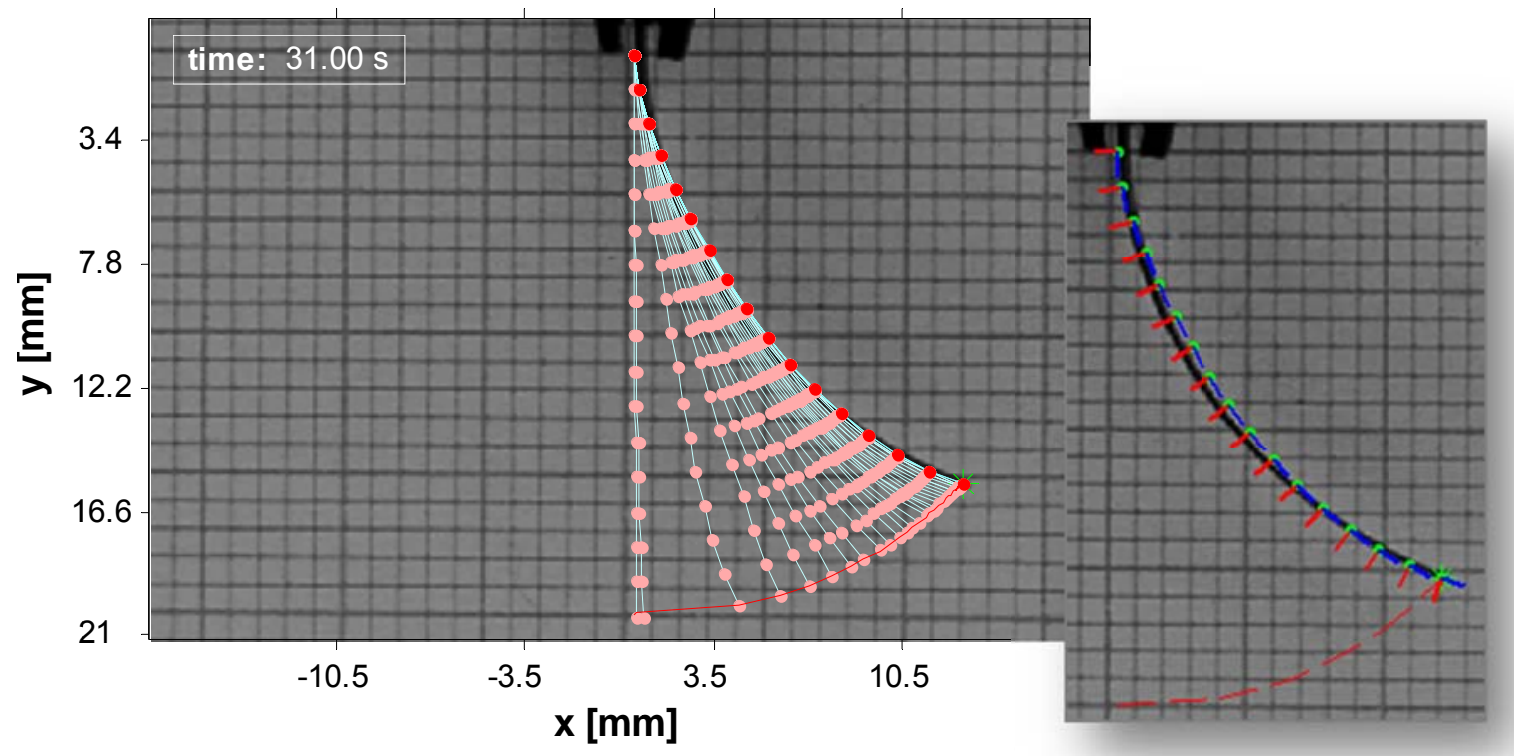

Fig.8. Shape correspondence between the 16-DoF hyper redundant model and the PPy-EAP actuator under $0.50 \mathrm{~V}$.

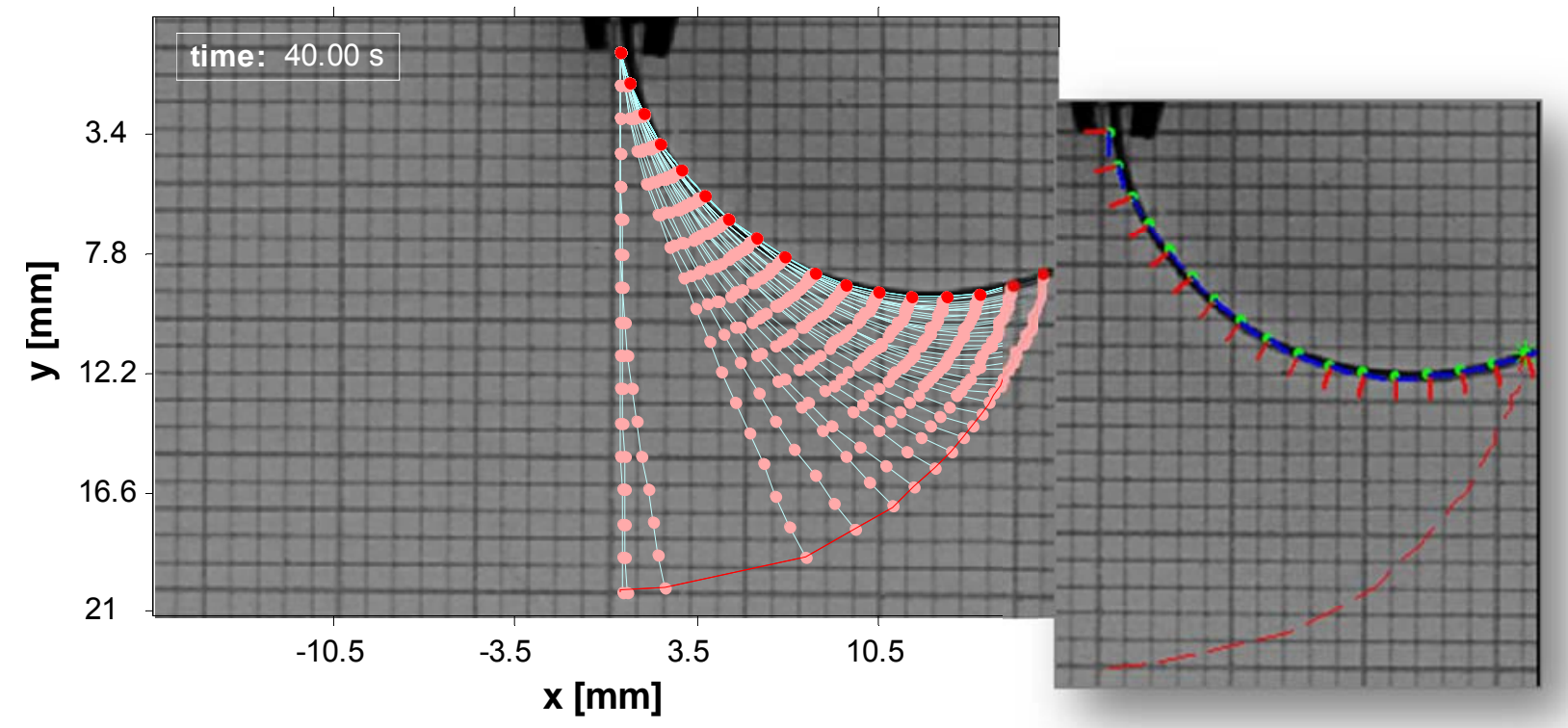

Fig.9. Shape correspondence between the 16-DoF hyper redundant model and the PPy-EAP actuator under $0.75 \mathrm{~V}$. 


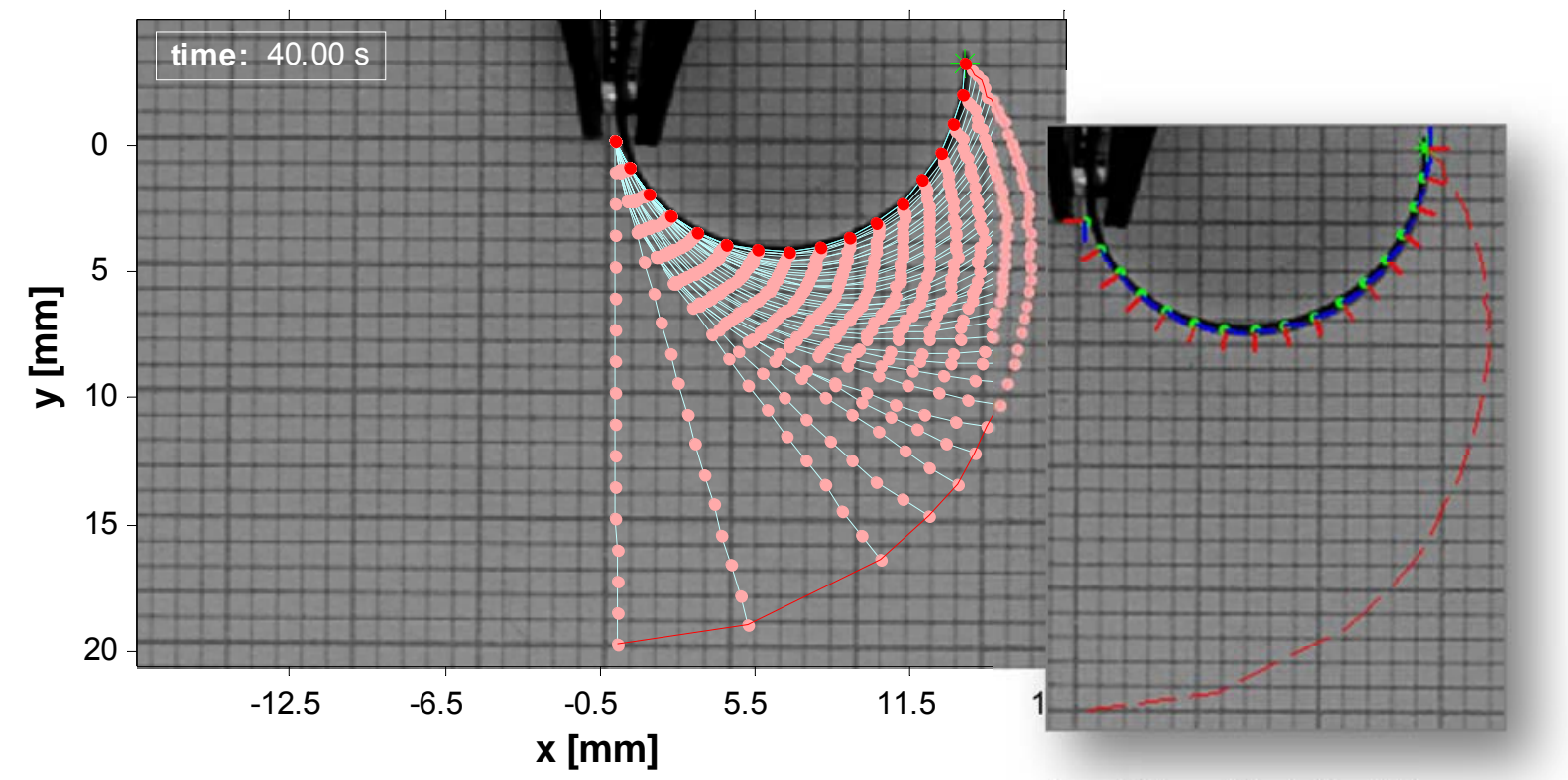

Fig.10. Shape correspondence between the 16-DoF hyper redundant model and the PPy-EAP actuator under $1.00 \mathrm{~V}$.

The RMS errors between the estimated and experimentally obtained tip coordinates are given for various electrical inputs in Table 1. The close correspondence between the experimental and numerical results demonstrates the efficacy of the AngleOPT method, and the constraints and conditions applied while solving the hyperredundant inverse kinematic model. The resulting RMS errors are possibly due to numerical truncations and experimental measurement errors. Also, the RMS errors calculated for the tip coordinates estimated by the Jacobian method described by Eqs. 19-21 are shown in Table 2 for a comparison with the results obtained from our optimization based approach, the AngleOPT. For a given end point coordinates $(X$, $Y$ ), the well-known Jacobian method is given by

$$
\begin{aligned}
& {[\dot{X}]=\mathcal{J}[\dot{\theta}]} \\
& {[\dot{\theta}]=\mathcal{J}^{\dagger}[\dot{X}]}
\end{aligned}
$$

where pseudo-inverse of the Jacobian matrix is described as

$$
\mathcal{J}^{\dagger}=\mathcal{J}^{\mathrm{T}}\left(\mathcal{J} \mathcal{J}^{\mathrm{T}}\right)^{-1}
$$

The RMS results indicate that the AngleOPT method is highly effective for the solution of the inverse kinematic problem of hyper-redundant systems. On the other hand, the Jacobian method cannot estimate the joint angles accurately, especially when the configuration increments are not small enough. One of the main reasons 
for this inaccuracy of the Jacobian method for the inverse kinematic solution of the hyper-redundant systems is the numerical instabilities associated with calculating the inverse Jacobian, especially in the vicinity of the singular positions [28]. In addition to this, the Jacobian method has no control over the calculated joint positions such that they result in a continuum body configuration at every instant. As articulated above, the AngleOPT method guarantees this configuration correspondence between the hyper-redundant model and the real system, the PPy-EAP actuator.

TABLE 1. RMS errors between experimentally obtained and estimated tip data under electrical inputs $0.25-1.0 \mathrm{~V}$ (method used for the inverse kinematic calculations is the AngleOPT).

\begin{tabular}{ccccc}
\hline \hline $\begin{array}{c}\text { RMS } \\
\text { axis }\end{array}$ & $\mathbf{0 . 2 5 V}$ & $\mathbf{0 . 5 V}$ & $\mathbf{0 . 7 5 V}$ & $\mathbf{1 . 0 V}$ \\
\hline $\boldsymbol{X}$ & 0.4511 & 0.7115 & 0.8456 & 0.4319 \\
$\boldsymbol{Y}$ & 0.6115 & 0.7829 & 0.4749 & 0.3846 \\
\hline \hline
\end{tabular}

TABLE 2. RMS errors between experimentally obtained and estimated tip data under electrical inputs $0.25-1.0 \mathrm{~V}$ (method used for the inverse kinematic calculations is the Jacobian method).

\begin{tabular}{ccccc}
\hline \hline $\begin{array}{c}\text { RMS } \\
\text { axis }\end{array}$ & $\mathbf{0 . 2 5 V}$ & $\mathbf{0 . 5 V}$ & $\mathbf{0 . 7 5 V}$ & $\mathbf{1 . 0 V}$ \\
\hline $\boldsymbol{x}$ & 5.2610 & 11.3015 & 5.8409 & 11.8415 \\
$\boldsymbol{y}$ & 1.2846 & 8.6389 & 23.3172 & 35.1489 \\
\hline \hline
\end{tabular}

In addition to estimating whole shape behaviour of the PPy-EAP actuator using the AngleOPT method, we include a parametric estimation method in the proposed methodology that this parametric model estimates the tip displacement of the PPyEAP actuator as a function of time for a given electrical input. Integrating this parametric model into the AngleOPT will result in a complete model estimating the whole shape of the actuator in real time for a given electrical input. We have chosen a quintic polynomial for the parametric model, as given below;

$$
\begin{aligned}
& X=A_{0}(V)+A_{1}(V) t+A_{2}(V) t^{2}+\cdots+A_{5}(V) t^{5} \\
& Y=B_{0}(V)+B_{1}(V) t+B_{2}(V) t^{2}+\cdots+B_{5}(V) t^{5}
\end{aligned}
$$


where $t$ is time, $A_{n}(V)$ and $B_{n}(V)$ are the input-voltage dependent coefficients.

This parametric model is valid for the PPy-EAP actuator with the dimensions of $20 \mathrm{x}$ $3 \times 0.17 \mathrm{~mm}$. However, the parametric model can be extended to estimate the tip displacements of different size PPy-EAP actuators by introducing the dimensions as another variable in the parametric model. Tip estimation results under a range of electrical inputs (0-1.0V) are given in Figures 11 and 12.

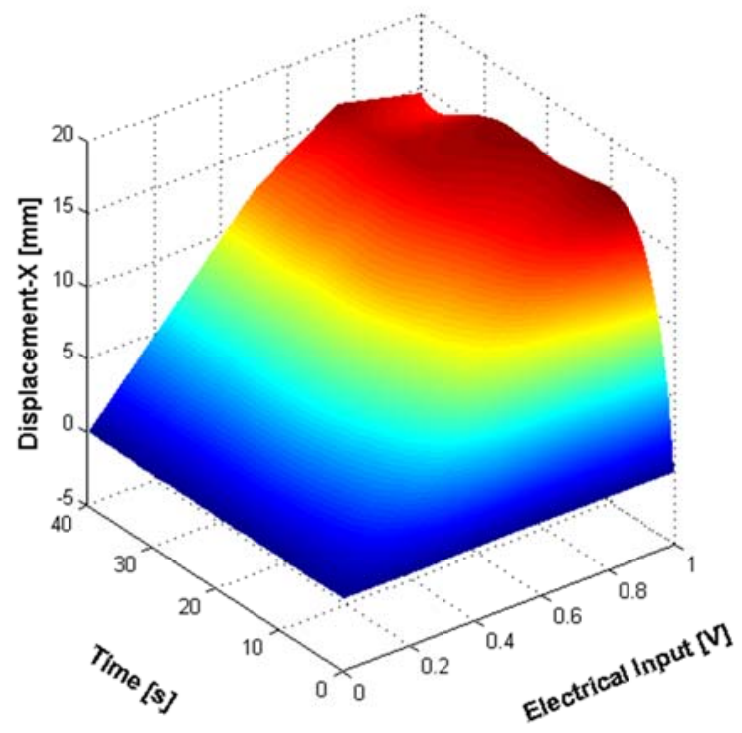

Fig.11. $X$ coordinate of the tip position estimation using the parametric model

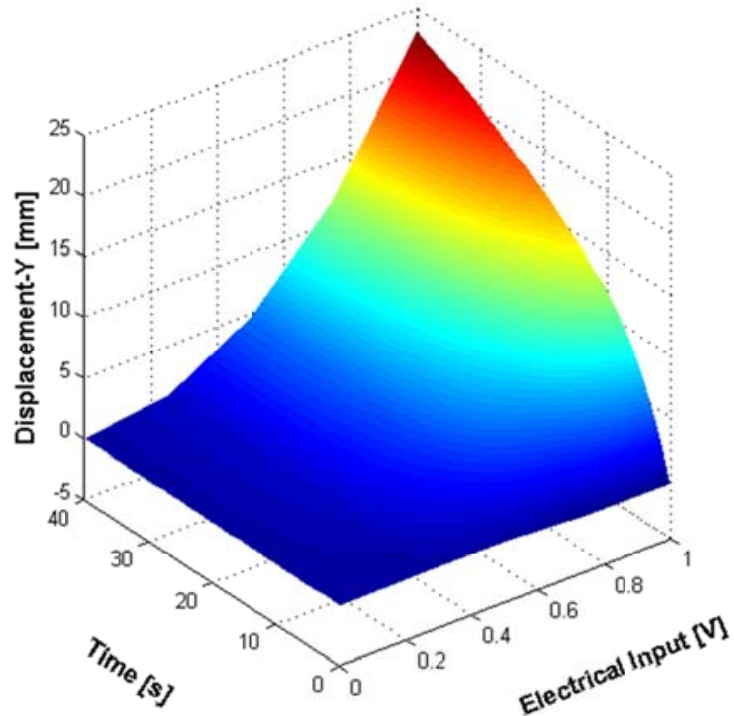

Fig.12. $Y$ coordinate of the tip position estimation using the parametric model 

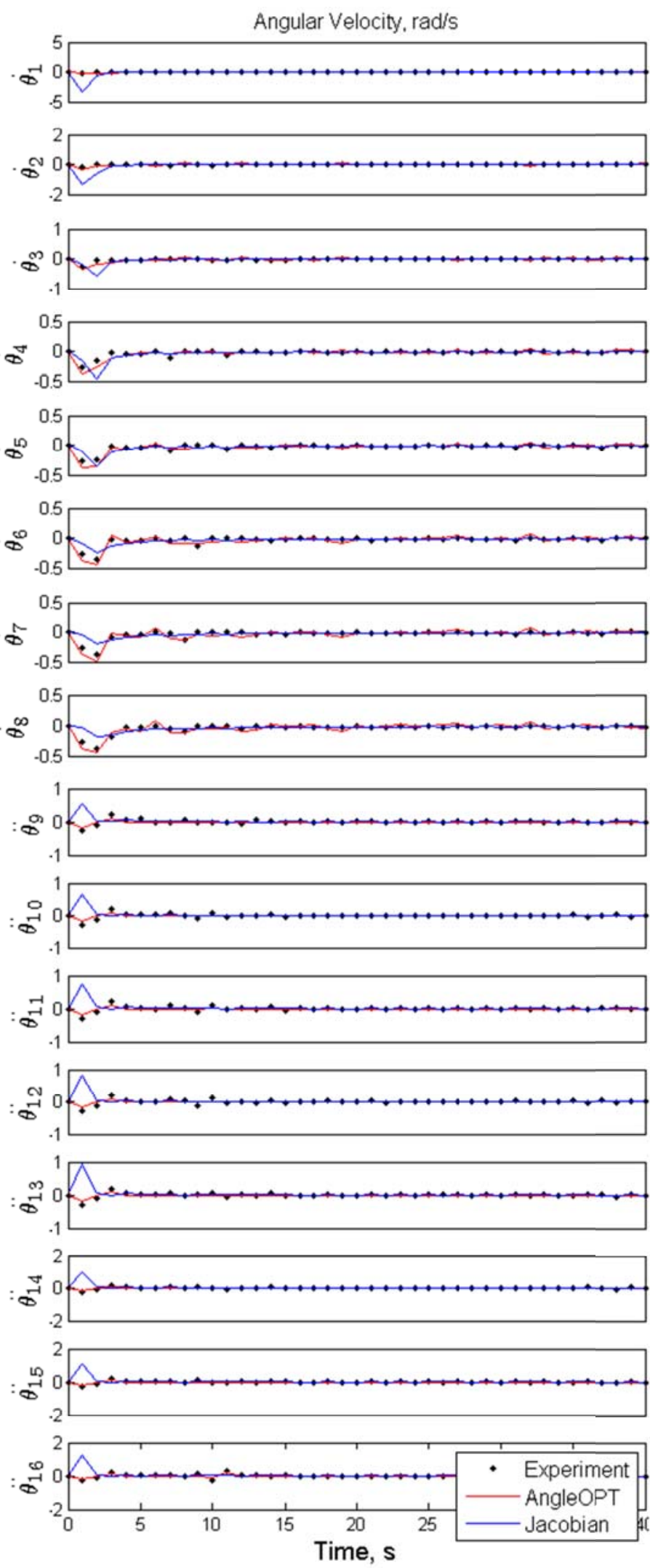

Fig.13. Angular velocity comparison for all joints of the 16-DoF model of the PPy polymer actuator (Color print).
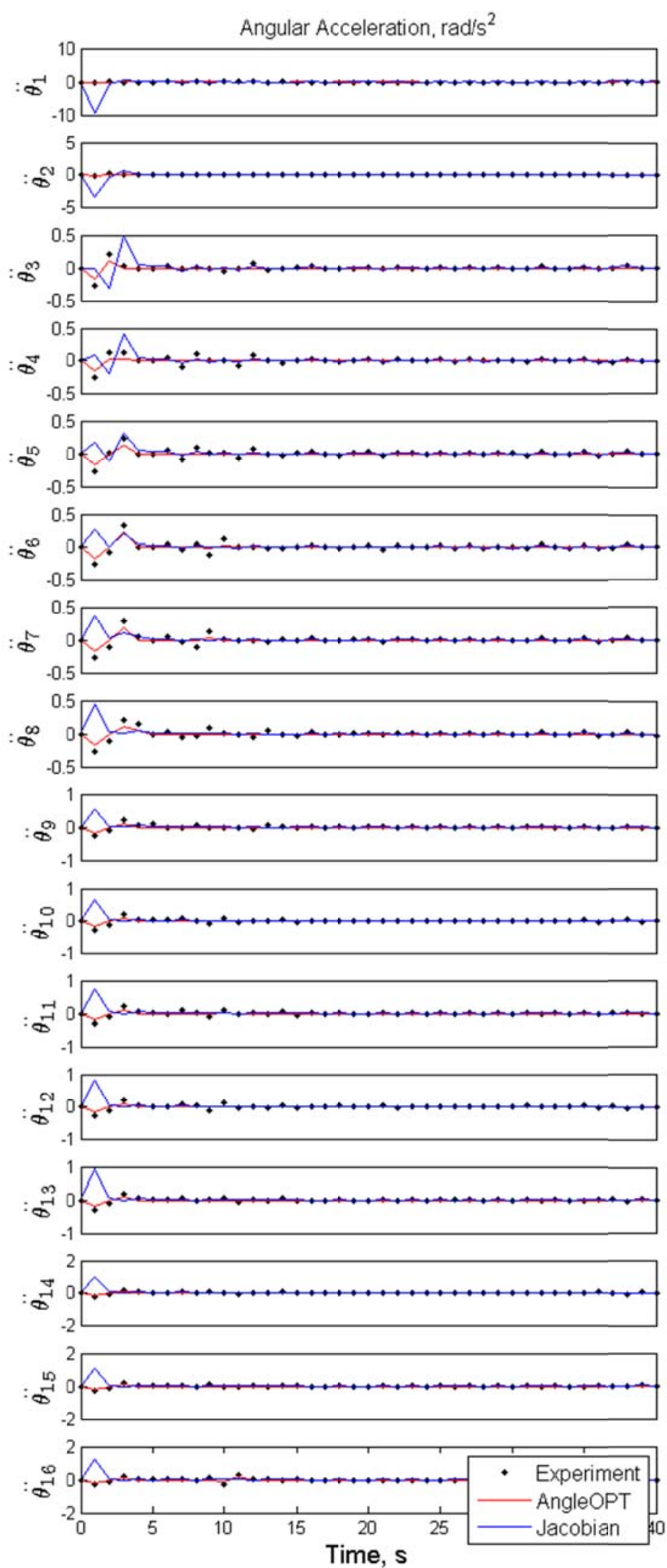

Fig.14. Angular acceleration comparison for all joints of the 16-DoF model of the PPy polymer actuator (Color print). 


\subsection{Estimation of Velocity and Acceleration}

We estimate the velocity and acceleration of the actuator tip point from the angular velocity and acceleration of all joints of the 16-DoF hyper-redundant model. We have used two methods to determine the velocity and acceleration of the PPy-EAP actuator; i) the AngleOPT method and ii) the Jacobian method. Required angular displacements for both methods are provided from the configuration estimation (AngleOPT) results.

As shown in Fig.13, the AngleOPT method is more effective than the Jacobian method in estimating the angular velocities of the hyper-redundant robotic manipulator model. The possible reasons are that the Jacobian method suffers from the numerical instabilities, problems associated with the matrix inversion and singularity problems as articulated above. The same methods are employed to estimate the joint angular accelerations for the 16-DoF hyper-redundant model, as shown in Fig.14. For the sake of brevity, angular velocity and acceleration estimations for $1.0 \mathrm{~V}$ electrical input only are shown in Figures 13 and 14 . The experimental velocity and acceleration data in Figures 13 and 14 are obtained from the variation of the angular displacements of the joints over time $\left(\Delta \theta_{i} / \Delta t_{i}\right)$, and the variation of the angular velocities of the joints over time $\left(\Delta \dot{\theta}_{\mathrm{i}} / \Delta \mathrm{t}_{\mathrm{i}}\right)$, respectively.

\subsection{Discussion}

2-DoF and 16-DoF hyper-redundant models of the PPy-EAP actuator are analysed and their inverse kinematic shape estimation problems are solved using the AngleOPT and the Jacobian methods. For the 2-DoF model, the AngleOPT method and the Jacobian method were compared with the analytical method. the AngleOPT method has performed as accurate as the analytical method. Although there was an error accumulation in the solutions from the Jacobian method, the results were still acceptable, but less accurate than the results from the AngleOPT method. For the 16-DoF hyper-redundant model, the AngleOPT method has been employed to obtain the inverse kinematic solutions. The main reasons for choosing the AngleOPT for the solution of the hyper-redundant model include (i) the AngleOPT method accurately calculates the joint angles/positions of the PPy-EAP actuator, (ii) it does not accumulates the estimation errors, (iii) the PPy-EAP actuator's physical boundaries and conditions can be considered for an improved configuration correspondence 
between the hyper-redundant model and the real PPy-EAP actuator, and (iv) the AngleOPT does not suffer from the singularity problem associated with the angular positions. As the AngleOPT is a non-linear constraint optimization method, the adaptive constraints and boundaries can be applied to obtain reasonably accurate inverse kinematic solutions. The adaptive boundary and constraints are updated at every instant while all configurations of the PPy-EAP actuator are estimated as a function of time. The 16-DoF hyper-redundant model solved with the AngleOPT method generates a quite well configuration correspondence with the real PPy-EAP actuator. As an extension of this work, we have established a dynamic model of the EAP actuator and identified its dynamic parameters experimentally such that the kinematic and dynamic models can be employed together to control the output displacement of the EAP actuators using a feedforward control method not requiring any external feedback data. With this in mind, an accurate kinematic model is needed to estimate the actuator configuration accurately for a given voltage input so that its positioning ability can be improved without using external sensors. It is not practical to use an internal or external sensor to measure the actuator displacement online for feedback control. The ideal solution is to have accurate models and invert these models to control the displacement output. This work is well-underway and will be reported in the near future.

\section{CONCLUSIONS and FUTURE WORK}

We have presented an efficient method to estimate the angular position, velocity and acceleration of the bending type ionic EAP actuators, which is treated as a hyperredundant soft robotic structure. The proposed method, which solves the inverse kinematic variables of the hyper-redundant model with $16-\mathrm{DoF}$, employs the AngleOPT as the solver. The advantage of the proposed method is that it can be applied to the solution of the inverse kinematic shape estimation problem of any DoF hyper-redundant and soft robotic structures including artificial muscles like EAP actuators. An image processing system has also been employed to obtain the tip coordinates of the PPy-EAP actuator experimentally. We have employed a parametric model to estimate the coordinates of the tip point of the actuator as a function of time for a given voltage input. This model complements the methodology to estimate the actuator's whole shape deflection. The AngleOPT also demonstrates a better performance when estimating the angular velocity and angular acceleration 
of the 16-DoF hyper-redundant model. Though the examples in the present paper are demonstrated for the PPy-EAP actuator (i.e. multi-layer laminated ionic EAP actuator), this method can be used to estimate the kinematic configuration and bending displacements of other EAP actuators. Estimating the whole kinematic configuration of continuum active structures based on EAP actuators can find places in biomedical applications such as a catheter or guiding a cochlear implant through a 3D spiral structure if they are designed for these tasks [32].

As future work, we will employ this nonlinear optimization method to solve the dynamic model of the PPy-EAP actuators to be treated as the hyper-redundant soft robotic structures. Also, the AngleOPT method will be extended to a 3-dimensional inverse kinematic problem (i.e. 3D shape forming rather than only bending in 2D plane) which includes a full representation of the electroactive polymer actuator with cross-section orientations including a twist along the EAP actuator length.

\section{Acknowledgement}

This work was supported in part by the ARC Centre of Excellence for Electromaterials Science (CE0561616), an ARC Discovery project (Grant No. DP0878931), and the University of Wollongong Research Council with a PhD scholarship for the first author.

\section{References}

[1] E. Smela, Conjugated polymer actuators for biomedical applications, Advanced Materials, 15 (2003), pp.481-494.

[2] G. Alici, G.M. Spinks, J.M.D. Madden, Y. Wu \& G.G. Wallace, Response Characterisation of Electroactive Polymers as Mechanical Sensors, IEEE/ASME Transactions on Mechatronics, 13 (2008), pp.187-196.

[3] Y. Wu, G. Alici, G.M. Spinks \& G.G. Wallace, Fast tri-layer polypyrrole bending actuators for high speed applications, Synthetic Metals, 156 (2006), pp.1017-1022.

[4] S. McGovern, G. Alici, V. Truong et al., Finding NEMO (novel electromaterial muscle oscillator): a polypyrrole powered robotic fish with real-time wireless speed and directional control, Smart Materials \& Structures, 18 (2009), p.095009.

[5] G. Alici, V. Devaud, P. Renaud \& G.M. Spinks, Conducting polymer microactuators operating in air, J. Micromechanics and Microengineering, 19 (2009), p.025017.

[6] G. Alici \& D. Gunderson, A bio-inspired robotic locomotion system based on conducting polymer actuators, IEEE/ASME International Conference on Advanced Intelligent Mechatronics, Singapore, 2009, pp.998-1004.

[7] R. Mutlu \& G. Alici, A multistable linear actuation mechanism based on artificial muscles, Journal of Mechanical Design, 132 (2010), p.111001.

[8] G. Alici \& N.N. Huynh, Performance quantification of conducting polymer actuators for real applications: A microgripping system, IEEE/ASME Transactions. Mechatronics, 12 (2007), pp. 73-84.

[9] S. John, G. Alici \& C. Cook, Frequency response of polypyrrole trilayer actuator displacement, Conference on Electroactive Polymer Actuators and Devices, 2008, 6927: 69271T. 
[10] S.W. John, G. Alici \& C. Cook, Validation of resonant frequency model for polypyrrole trilayer actuators, IEEE-ASME Transactions on Mechatronics, 13 (2008), pp.401-409.

[11] S.W. John, G. Alici \& C. Cook, Towards the Position Control of Conducting Polymer Trilayer Bending Actuators with Integrated Feedback Sensor, IEEE/ASME International Conference on Advanced Intelligent Mechatronics, 1-3 (2009), pp.65-70.

[12] G. Alici, An effective modelling approach to estimate nonlinear bending behaviour of cantilever type conducting polymer actuators, Sensors And Actuators B-Chemical, 141 (2009), pp.284-292.

[13] S.W. John, G. Alici \& C. Cook, Inversion-based feedforward control of polypyrrole trilayer bender actuators, IEEE-ASME Transactions on Mechatronics, 15 (2010), pp.149-156.

[14] Y. Yekutieli et al., Dynamic model of the octopus arm. I. Biomechanics of the octopus reaching movement, Journal of Neurophysiology, 94 (2005), pp.1443-1458.

[15] F. Boyer, M. Porez \& W. Khalil, Macro-continuous computed torque algorithm for a threedimensional eel-like robot, IEEE Transactions on Robotics, 22 (2006), pp.763-775.

[16] M. Hannan \& I.D. Walker, Analysis and initial experiments for a novel elephant's trunk robot, Proc. Int. Conf. on Intelligent Robots and System , 1 (2000), pp.330-337.

[17] M. Hannan \& I.D. Walker, Novel kinematics for continuum robots, In: 7th Int. Symp. Advances in Robot Kinematics, 2000, pp.227-238.

[18] M. Hannan \& I.D. Walker, Kinematics and the implementation of an elephant's trunk manipulator and other continuum style robots, Journal of Robot Systems, 20 (2003), pp.45-63.

[19] K Suzumori, S likura \& H Tanaka, Development of flexible microactuator and its application to robotic mechanisms, IEEE International Conference on Robotics and Automation, 1(1991), pp.1622-27.

[20] S. Hirose \& M. Mori, Biologically inspired snake-like robots, Proceedings of the 2004 IEEE International Conference on Robotics and Biomimetics, 2004, pp. 22-26.

[21] H. Mochiyama \& H. Kobayashi, Shape correspondence between a spatial curve and a manipulator with hyper degrees of freedom, Proc. IEEE/RSJ Int. Conf. Intelligent Robots and Systems, 1 (1998), pp.161-166.

[22] G.S. Chirikjian \& J.W. Burdick, Kinematically optimal hyper-redundant manipulator configurations, IEEE Transactions on Robotics and Automation. 11(6), 1995, pp.794-806.

[23] G.S. Chirikjian, Hyper-redundant manipulator dynamics: A continuum approximation, Advanced Robotics, 9(3), 1995, pp.217-243.

[24] G.S. Chirikjian, Inverse kinematics of binary manipulators using a continuum model, Journal of Intelligent Robotic Systems, 19 (1997), pp.5-22.

[25] Ravi, V. C., Rakshit, S., and Ghosal, A., "'Redundancy resolution using tractrix -- simulations and experiments", Trans. of ASME, Journal of Mechanisms and Robotics, Vol. 2, pp. 031013-1, 2010.

[26] Sreenivasan, S., Goel, P., and Ghosal, A., “'A real-time algorithm for simulation of flexible objects and hyper-redundant manipulators", Mechanism and Machine Theory, Vol. 45, pp. 454466, 2010.

[27] R. Mutlu \& G. Alici, Artificial muscles with adjustable stiffness, Smart Materials and Structures, 19 (2010), p.045004.

[28] R. Mutlu, G. Alici \& W. Li, Kinematic modeling for artificial flagellum of a robotic bacterium based on electroactive polymer actuators, IEEE/ASME International Conference on Advanced Intelligent Mechatronics, 2011, pp.440 -445.

[29] G. Alici, "An Inverse Position Analysis of Five-bar Planar Parallel Manipulators", Robotica, 20:2, pp.195 -- 201, March 2002.

[30] V.A. Toponogov, Theory of curves in three-dimensional Euclidean space and in the plane, in Differential Geometry of Curves and Surfaces: A Concise Guide, Birkhäuser, Boston, 2006, pp.1-63.

[31] J.J. Craig, Inverse manipulator kinematics, in Introduction to Robotics: Mechanics and Control, 3rd ed., Pearson Prentice Hall, NJ, 2005, pp.101-134.

[32] G.M. Spinks, G. Alici, S. McGovern, B. Xi \& G.G. Wallace, Conjugated Polymer Actuators: Fundamentals, in Biomedical Applications of Electroactive Polymer Actuators: John Wiley \& Sons, 2009, pp. 193-227. 


\section{Appendix A: Setting Constraints and Boundaries}

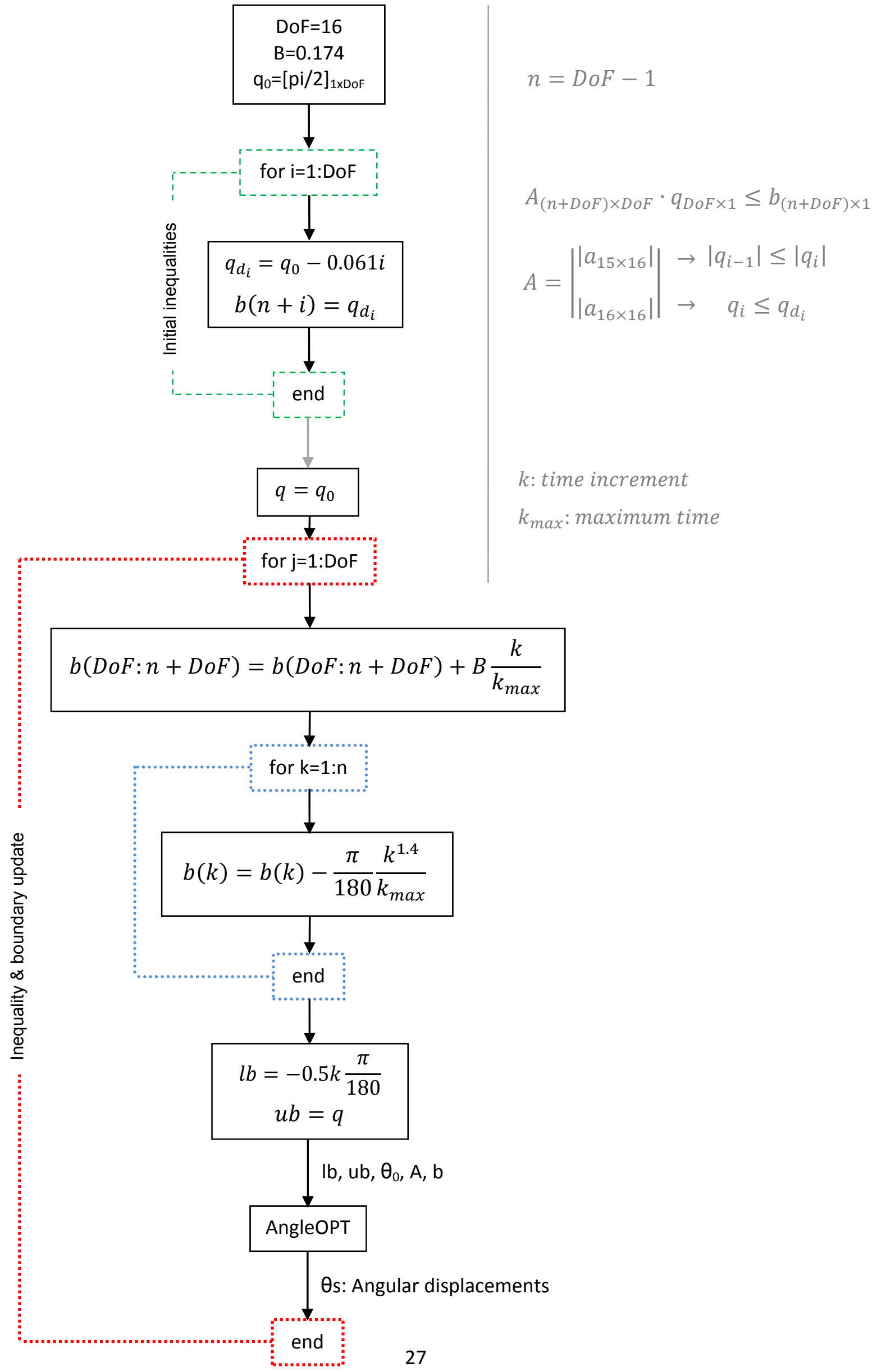


We propose this algorithm to determine the boundary conditions and constraints for the joint angles, which are adaptively changed $\mathrm{n}$ order to reflect physical conditions of the real PPy-EAP actuator on the solutions searched. First, the initial inequality values, $q_{d_{i}}$, for joint angles are assigned. Then these inequalities are changed by time using the coefficient $B$. These inequalities constrain the joint angles to certain values depending on the electrical input. Also, a continuous relationship between neighbour joint angles, $\left|q_{i-1}\right| \leq\left|q_{i}\right|$ is assigned to ensure that the calculated joint angles closer to the free end of the PPy-EAP actuator are larger than the joint angles closer to the fixed end. $\mathrm{k}$ and $k_{\max }$ are the time increment and the final time, respectively that at each time increment, the boundaries/constraints are adaptively changed as the real PPy-EAP actuator's bending configuration changes. 